\title{
Chronic iron deposit and left ventricular remodeling in reperfused STEMI patients
}

\author{
Heerajnarain Bulluck ${ }^{1,2^{*}}$, Steven K White ${ }^{2}$, Stefania Rosmini ${ }^{2}$, Amna Abdel-Gadir ${ }^{2}$, Anish N Bhuva ${ }^{2}$, \\ Thomas A Treibel ${ }^{2}$, Marianna Fontana ${ }^{2}$, Patricia Reant ${ }^{2}$, Manish Ramlall ${ }^{1,2}$, Ashraf Hamarneh ${ }^{1,2}$, Alex Sirker $^{3}$, \\ Anna S Herrey ${ }^{2}$, Charlotte Manisty ${ }^{2}$, Peter Kellman ${ }^{4}$, James Moon², Derek J Hausenloy ${ }^{1}$
}

From 19th Annual SCMR Scientific Sessions

Los Angeles, CA, USA. 27-30 January 2016

\section{Background}

After reperfused ST-segment elevation myocardial infarction (STEMI), infarct zone microvascular obstruction (MVO) and intramyocardial hemorrhage are associated with left ventricular (LV) remodeling. We wanted to understand what happened to areas of haemorrhage and observe its resolution/persistence and any role it may play in remodeling.

\section{Methods}

48 STEMI patients underwent CMR imaging at $1.5 \mathrm{~T}$ (Siemens Avanto) at $4 \pm 2$ days post-PPCI and 40 completed a follow-up scan at $5 \pm 2$ months. Left ventricular (LV) short-axis native T1 (MOLLI), T2 and T2* maps were acquired. MVO was indicated by a hypo-intense core on LGE images. A hypo-intense core on $\mathrm{T} 2{ }^{*}$-maps with a $\mathrm{T} 2 *<20 \mathrm{~ms}$ was used for IMH (acutely) or chronic iron deposit (at follow-up). Mean segmental T2 and T1 values were obtained using CVI42 (Calgary, Canada). LV remodeling was defined as a $20 \%$ increase in LV end-diastolic volume on the follow-up scans.

\section{Results}

Acute scan

MVO was present in $63 \%$ patients. T2* maps was used as the reference standard (17\% excluded due to motion and breathing artifacts rendering then uninterpretable). T1 and T2-mapping performed equally well in detecting the presence of IMH on the acute scan (T1: AUC 0.86 [95\%CI 0.72-0.99], T2: AUC 0.86 [95\%CI 0.74-0.99]; $\mathrm{P}=0.94$ ) (Figure 1). 29/30(95\%) patients with MVO had evidence of IMH (T2*<20 ms).

The Hatter Cardiovascular Institute, University College London, London, United Kingdom

Full list of author information is available at the end of the article

\section{Follow scan}

8/40(20\%) patients had evidence of LV remodeling on the follow-up scan. All patients who developed LV remodeling had MVO and IMH acutely, compared to $60 \%$ in patients without $\mathrm{LV}$ remodeling $(\mathrm{P}=0.04)$. $37 \%$ of $\mathrm{T} 2$ * maps were excluded on the follow-up scan. 13/15(87\%) of patients had evidence of chronic iron deposition within the infarct core ( $\mathrm{T} 2 * 15 \pm 2 \mathrm{~ms})$. In these patients, $\mathrm{T} 1$ and T2 values were elevated within the areas of late gadolinium enhancement (Figure 2) and were higher than those without iron deposit.

\section{Conclusions}

$\mathrm{T} 1$ and T2-mapping can detect hemorrhage.

Acute hemorrhage becomes chronic and is still detectable at 5 months

Infarct T2 normalizes usually by 3-6 months in most patients - but around chronic iron the T2 remains high suggesting the iron may be a source of ongoing inflammation.

\section{Authors' details}

'The Hatter Cardiovascular Institute, University College London, London, United Kingdom. ${ }^{2}$ Cardiac Imaging, Barts Heart Centre, London, United Kingdom. ${ }^{3}$ Barts heart Centre, London, United Kingdom. ${ }^{4} \mathrm{NIH}$, Bethesda, $\mathrm{CA}$, USA.

Published: 27 January 2016

doi:10.1186/1532-429X-18-S1-P230

Cite this article as: Bulluck et al: Chronic iron deposit and left

ventricular remodeling in reperfused STEMI patients. Journal of

Cardiovascular Magnetic Resonance 2016 18(Suppl 1):P230. 

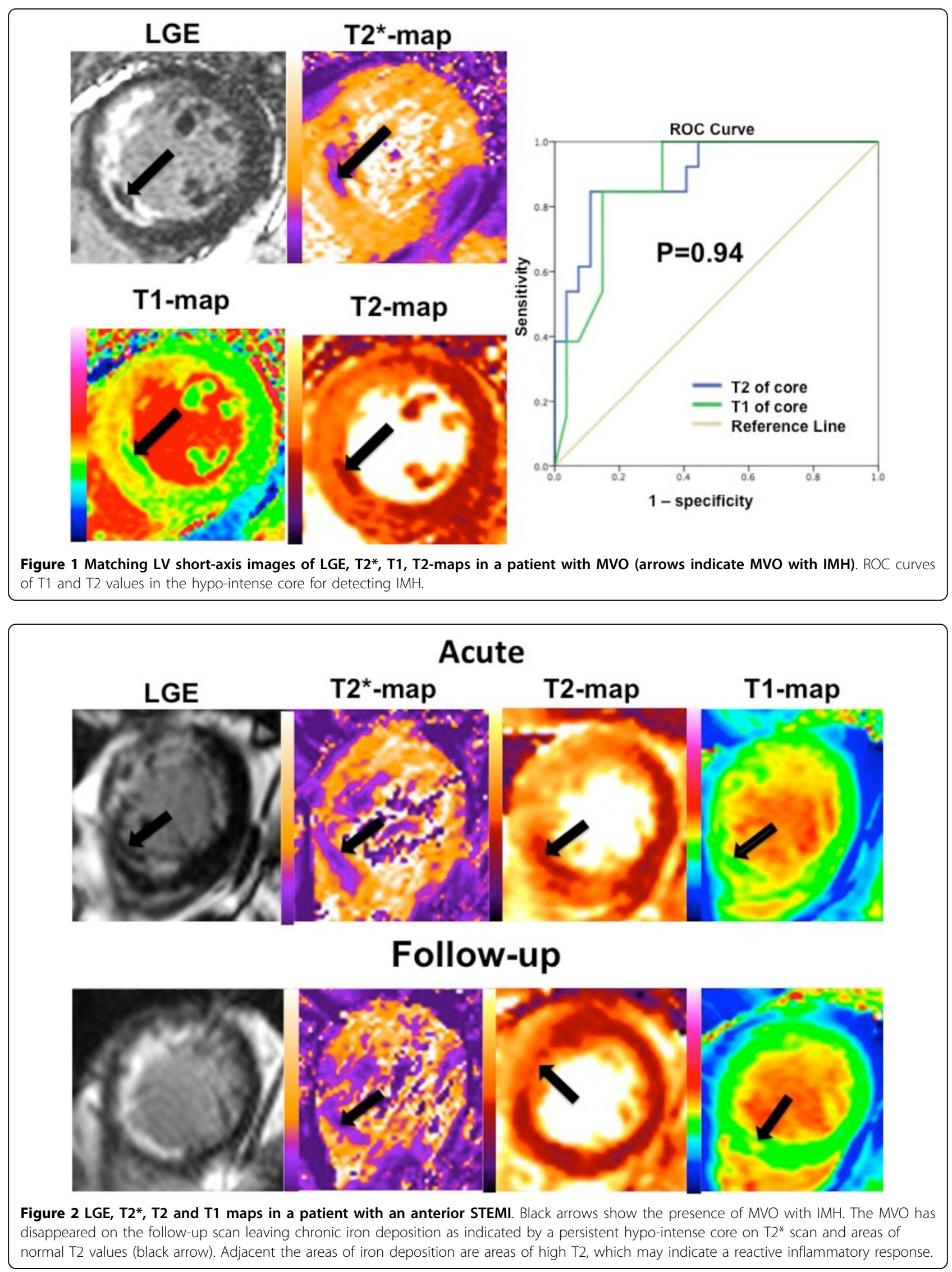